\title{
Drug Repositioning: Discovering New Virtues in The Past
}

\section{Maruthi Prasad Palthur*}

Indigene Pharmaceuticals, Inc., Westborough, MA 01581, USA

The past is generally considered as a repository of knowledge and experience. From time to time, we look into the past to find solutions to the challenges of the future. For the most part, the history will not give us the answers directly. However, it could help us better understand the results of past experiences, and does play a pivotal role in making right decisions to solve the current and future challenges.

Despite of heavy investments in biomedical research, the unconvincing performance of the pharmaceutical industry has become a global concern in the recent years, and is currently acknowledged as a universal challenge. Pharmaceutical industry is currently facing enormous criticism for its reduced efficiency, declining innovation, escalating developmental costs, competition from generics, diminished pipeline, patent expirations and safety concerns. Today's drug discovery and development is time consuming and is universally acknowledged to be costly and failure-prone process. Productivity has continued to decline and the rate of new drug development has changed little in the recent years.

As serendipitous discoveries diminish, along with the productivity of current approaches, there seems to be a clear need for a change in the current paradigms to address these challenges. As research and innovation are indispensable for improving health, unorthodox thinking appears to be essential in overcoming these persistent challenges Historically, scientific fraternity and industry has responded by adopting a range of initiatives and technology to foster innovation and to pursue any transformative approaches. As an enterprise and a tactic, a deliberation for historic wealth of natural products and approved drugs pharmacological information has been emphasized to generate drugs and drug leads. Despite the fact that those natural products and their derivatives have been historically invaluable source of drug leads, a diminished interest in natural products drug discovery is observed. In addition, sophisticated technologies such as combinatorial chemistry and high-throughput screening that have taken the center stage in drug discovery failed to meet their expectations. Coupled with regulatory challenges and health care costs, there seems to be a compelling case and renewed interest towards approved drugs as a source of potential drug leads or new indications. This sort of initiative, goes by a diversity of terms-drug recycling, drug repurposing, drug repositioning, drug rescuing, drug rediscovery-is a young discipline of finding innovative ways to position approved drugs or abandoned clinical candidates in new disease areas. Drug rescue and repurposing are such two divergent strategies. Drug "rescue" refers to research involving small molecules and biologics whose development was abandoned before they could be approved by the regulatory authorities. "Repurposing" refers to studying small molecules and biologics approved to treat a disease or condition to investigate if they are safe and effective for treating other diseases. Approved drugs and many abandoned compounds for reasons other than safety, already have been tested in humans, and hence convincing information generally exists on their pharmacology, formulation, dosing and potential toxicity.

Logically speaking, repurposing obviously should yield a risk reduction business model, because it is built upon previous research and development efforts. Theoretically, repurposed drugs can enter clinical testing more rapidly, at less cost, and can optimize the potential value of a therapeutic drug. Repurposing is not new to the pharmaceutical industry-small and large companies used conventional life-cycle management approaches often to extend drug use into new indications as a measure protect or broaden the brand value. However, the current focus on repurposing is fueled by divergent key drivers, including key patent expirations, advances in science and technology, emergence of proprietary discovery engines, declined innovation, healthcare cost concerns, and support from Government initiatives and public funding.

As a result of the convergence of several market and economic forces, drug repositioning has become a mainstream drug development strategy for major pharmaceutical companies In the past, pharmaceutical companies have sometimes been reluctant for repurposing, and it was not extensively practiced owing to the concerns of potentially endangering the established indication by revealing safety or other issues. Few other reasons quoted in this context are, perceived low economic incentive of already off-patent drug, short regulatory market exclusivity for a generic repurposed drug, likely to compete with low-cost generics prescribed off-label, misalignments of novel indication with business strategy, diminutive potential market, and intense focus on new chemical entity discovery. However, only recently pharmaceutical companies adopted drug repositioning as a core research and development strategy. In addition, a rapid emergence of repurposing companies, proprietary repurposing discovery engines and repurposing computational platforms is observed. Additionally, support from Government initiatives and public funding bestowed a tremendous boost for repurposing.

In the past few years, government agencies, academic researchers, and the pharmaceutical industry have collaborated to shift drug repositioning from a serendipitous result to a comprehensive and rational agenda. European project PONTE, and initiatives of National Center for Advancing Translational Sciences (NCATS) are acknowledged as key initiatives in this regard. PONTE is a European project standing for 'Efficient Patient Recruitment for Innovative Clinical Trials of Existing Drugs to other Indications'. PONTE, aims at providing a platform following a service oriented architecture and semantic approach that will offer automatic intelligent identification of patients eligible to participate within well-specified clinical trials for drug repositioning with specific focus on mitigating patient safety risks, reducing clinical trial costs and improving clinical trial efficacy. Administered by NCATS, which is part of the National Institutes of Health, the 'Discovering New Therapeutic Uses for Existing Molecules' program aims to spur the drug development process by finding new uses for compounds that already have cleared several key steps in the development process, providing scientists with a strong head-start in this complex and time-consuming process. Through this innovative collaboration, several pharmaceutical companies have agreed to make some of their high-quality compounds available for research. The

${ }^{*}$ Corresponding author: Maruthi Prasad Palthur, Indigene Pharmaceuticals, Inc., Westborough, MA 01581, USA, E-mail: mpalthur@gmail.com

Received April 08, 2013; Accepted April 10, 2013; Published April 14, 2013

Citation: Palthur MP (2013) Drug Repositioning: Discovering New Virtues in The Past. Pharmaceut Reg Affairs 2: e121. doi:10.4172/2167-7689.1000e121

Copyright: (c) 2013 Palthur MP. This is an open-access article distributed under the terms of the Creative Commons Attribution License, which permits unrestricted use, distribution, and reproduction in any medium, provided the original author and source are credited. 
NCATS Pharmaceutical Collection (NPC), also known as the NIH Chemical Genomics Center (NCGC) Pharmaceutical Collection, is a comprehensive, publicly accessible collection of approved and investigational molecular entities for high-throughput screening that provides a valuable resource for both validating new models of disease and better understanding the molecular basis of disease pathology and intervention. NCATS currently possesses 2,500 , as well as about 1,000 additional investigational molecular entities, as part of its screening collection. The potential seems to be compelling and may fill the current innovation gap.

Repurposing is not free from challenges; some challenges are unique to this development approach, while others are common to any form of drug development. Repurposing unarguably is drug development, and is not risk-free. Despite of identifying good drug prospects, and existing data, repurposed drug candidates have the potential to fail in late-stage clinical trials and even after reaching the market. A repositioned drug has the same chances of success or failure as an original drug in meeting efficacy end points. Developers still have to fulfill regulatory requirements depending on the extent of change, and its new indication. Repositioning strategy is neither a direct path to assured success, nor going to substitute conventional drug discovery, however it may augment certain aspects of it. Achieving market exclusivity, a combination of intellectual property and regulatory exclusivity is assessed as a key determinant for commercial success of a repositioned drug. Cautious consideration is recommended to the potential generic competition in view of patent and regulatory exclusivity. However, orphan drug exclusivity and pediatric exclusivity in addition to the other regulatory exclusivities in some cases may offer an attractive incentive.

Although repurposing efforts have been underway for more than a decade now, it is difficult to make out any immense success stories from this hypothesis. However, most successful stories of repositioning are attributed to serendipity and not to a strict systematic analysis. Substantial evidence in support of this hypothesis is just mounting up, and so far it is inconclusive. However, few examples continue to maintain the hope for this hypothesis intact, for example, NIH initiation of phase I clinical trial to evaluate the safety and effectiveness of cyclodextrin as a potential therapy for Niemann-Pick type C1 by repurposing cyclodextrin, normally used as an inactive ingredient in certain formulated drug products.
Repositioning can mean new life for shelved or abandoned drugs, and it helps to fully explore the indications of marketed drugs and clinical candidates. The paradigm shift around drug repositioning is at a key transitional point, and may impart a much needed change in the current business models. The prospect for drug repositioning seems to be compelling, and its potential to bridge the innovation gap cannot be ruled out. The expansion of new resources, approaches, and technologies will increase the scope of successful repositioning, and may become more of a consideration for pharmaceutical research. Like its preceding drug developmental strategies the nature and scope of will evolve hopefully, yielding a much needed breakthrough. Regardless, starting with drugs of credible clinical experience may accommodate the interests of both industry and nonprofit groups. Industry perceives an opportunity for proceeds, and nonprofit groups perceive a resourceful approach to treat neglected diseases and address unmet medical needs.

However, streamlining collaboration, improvement in sharing of resources, expertise pooling from diverse fields, enhanced focus on integration of regulatory and scientific information, encouragement for academic drug discovery, integration of discovery technology platforms, and development of a more streamlined regulatory process worldwide, are among the prospective approaches that may progress the process of repurposing.

Despite of its failures the pharmaceutical industry has evolved and made tremendous progress in the past, and guided the emergence of new technologies and approaches. Revisiting approved drugs for new indications, and reinvestigating drug candidates for potential new therapeutic applications are the outcomes of this evolution. Drug repositioning that is well designed and executed offers a significant advantage over traditional drug development, and presents a compelling approach to delivering new treatments for a greater range of patients. The scope of drug repurposing can span from obvious, to the progressive innovation. However, repurposing unarguably is still drug development with obligations to fulfill regulatory requirements depending on the extent of change, and is not risk-free. Repositioning strategy is neither a direct path to assured success, nor going to substitute conventional drug discovery, however it may augment certain aspects of it. 